Meta

Journal des tradlucteurs

Translators' Journal

\title{
Faut-il réclamer ?
}

\section{Jean-Paul de Grandpré}

Volume 12, numéro 2, juin 1967

URI : https://id.erudit.org/iderudit/002498ar

DOI : https://doi.org/10.7202/002498ar

Aller au sommaire du numéro

Éditeur(s)

Les Presses de l'Université de Montréal

ISSN

0026-0452 (imprimé)

1492-1421 (numérique)

Découvrir la revue

Citer ce document

de Grandpré, J.-P. (1967). Faut-il réclamer ? Meta, 12(2), 51-56.

https://doi.org/10.7202/002498ar

Ce document est protégé par la loi sur le droit d'auteur. L'utilisation des services d'Érudit (y compris la reproduction) est assujettie à sa politique d'utilisation que vous pouvez consulter en ligne.

https://apropos.erudit.org/fr/usagers/politique-dutilisation/
Cet article est diffusé et préservé par Érudit.

Érudit est un consortium interuniversitaire sans but lucratif composé de l'Université de Montréal, l'Université Laval et l'Université du Québec à Montréal. Il a pour mission la promotion et la valorisation de la recherche. https://www.erudit.org/fr/ 


\section{FAUT-IL RÉCLAMER?}

Dans notre pays bilingue, on traduit a cat par un chat, ce qui est bien; on traduit parfois a translator par un transcripteur, ce qui est parfois le cas; et dans les assurances, lorsqu'il faut rendre le mot claim, on dit «réclamation », ce qui est un calque de transcripteur. Un anglicisme tenace, qui a jusqu'à ce jour résisté aux condamnations, qui a la vie dure comme Raminagrobis lui-même. $\AA$ A tous moments

1. Jean Brun, Dictionnaire de la radio, Paris, Albin Michel, 1949, p. 286. 
on nous le jette aux jambes: on fait des réclamations, on en calcule, on en paie, et sans doute les réclamants en dépensent-ils. Puis il y a eu le dernier de la lignée, « l'agent de réclamations ».

Tout en souhaitant que ce félin ne vive pas ses sept vies, demandons-nous pourquoi les rédacteurs et les traducteurs ont tant de mal à s'en débarrasser. Pudeur du mot juste? Influence des textes officiels ? Cette dernière, il faut le dire, est aussi forte que celle de tous nos comités de linguistique réunis. Songeons que si, il y a deux ans, l'administration nous avait proposé le mot expert au lieu d'agent de réclamations, ce vocable serait déjà d'usage courant; que si elle avait adopté plan de pension plutôt que régime de rente, cette dernière expression serait encore inconnue du public. Il y a aussi le fait que claim est un faux ami. La ressemblance verbale entre claim et réclamation est grande, et de plus, l'une des acceptions de claim correspond effectivement au sens de réclamation.

Voilà donc qui nous dicte de bien distinguer entre les sens de ces deux mots. Selon Koessler et Derocquigny, to claim peut signifier réclamer, mais aussi demander, élever des prétentions. Si ces deux dernières expressions sont assez faibles, en revanche, réclamer est un verbe fort. Deux des sens que lui prête le dictionnaire Hatzfeld et Darmesteter, nous intéressent: a) Exiger ce qu'on nous doit. Exemples: Réclamer sa part d'héritage - L'enfant naturel reconnu ne pourra réclamer les droits d'enfants légitimes (Code civil français, art. 338) - Réclamer en vertu du droit d'extradition un criminel réfugié dans un pays étranger. b) Demander quelque chose avec insistance. Exemples: Il (Luther) réclame le concile ... comme le seul remède des maux de l'Église (Boss.) - Il a reçu les soins que réclamait son état - L'amitié réclame une confiance mutuelle. Pour Koessler et Derocquigny, réclamer se traduit par to demand, lequel signifie demander comme un droit, péremptoirement, et Bénac note de son côté que réclamer enchérit sur demander.

Ces précisions mettent en lumière la valeur du mot à l'étude. L'emploi de ce verbe conviendrait dans le cas d'une situation à caractère frustrant: le résultat, par exemple, d'une promesse lâchement formulée, ou, chez l'assureur, d'une réputation de mauvais payeur. Les choses se passent-elles ainsi dans le commerce des assurances?

Dans le contrat d'assurance, la promesse est formelle, et l'obligation contractuelle, clairement établie. On trouve habituellement en première page des contratsvie, une phrase qui se lit à peu près comme suit: The $X$ Life Insurance Company agrees to pay on receipt of due proof of the death of the Insured the amount of insurance as a death benefit to the beneficiary (Principles of Life Insurance, p. 169). Dans les autres branches d'assurance l'engagement n'est pas moins catégorique. Sans doute la Compagnie sent-elle le besoin de se protéger contre la fraude et les manœuvres dolosives, et parfois d'exclure certains risques dont la réalisation est de constatation difficile. Mais ces précautions confirment la règle.

La pratique des sociétés d'assurance est d'ailleurs révélatrice sous ce rapport. Elles se font un point d'honneur de verser les sommes assurées dans les plus courts délais. Certaines de leurs formules publicitaires s'inspirent même de cette diligence. Nous tirons de l'un des volumes mis à la disposition des agents la remarque suivante: Insurance companies often take the responsibilities of arranging settlements directly with the beneficiaries or estates and take pride in the speed and efficiency with which such settlements are made. Signalons enfin le cas d'une compagnie qui 
a rédigé à l'intention des ayants droit une demande de règlement qui commence ainsi: You are authorized and requested to pay the sum of ... N'est-ce pas là une invitation à faire état dans le vocabulaire d'une attitude qu'on ne peut mettre en doute dans les faits?

L'obligation de l'assureur naît du jour où le risque se réalise, et celles de l'assuré ou de ses ayants droit prennent fin en même temps. Il ne reste alors à ceux-ci qu'à demander le paiement des sommes assurées. Pourquoi, en juste langue, devraient-ils les réclamer puisque ces sommes leur sont dues de plein droit et qu'elles leur sont remises sur demande?

Le caractère d'anglicisme sémantique de réclamation et l'automatisme de la garantie d'assurance étant respectivement établis, explorons maintenant les sens du mot claim. Ce terme a dans les assurances diverses acceptions. Elles sont sans doute apparentées, mais assez distinctes pour commander des nuances dans la traduction. Claim peut signifier: 1) le fait qui constitue la réalisation du risque: la mort, la malcidie, l'incapacité de travail, etc.; 2) la demande de la somme stipulée au contrat et cette somme elle-même; 3) le titre ou le droit à l'assurance; 4) la revendication, lorsq'u'elle s'impose. À ces différents sens correspondent en français universel des expressions consacrées.

\section{PREMIER SENS}

La condition prévue au contrat se réalise. Cet événement s'appelle le sinistre. (Cf. Hatzfeld et Darmesteter: Perte, dommage que subissent les assurés.) C'est l'expression qu'utilisent les auteurs et rédacteurs francophones: Il peut être difficile de connaître l'état mental de l'assuré au moment du sinistre (Maurice Fauque, les Assurances) - Le risque est l'événement aléatoire dont la réalisation constitue le sinisite (Ibid.) - Ils (les ayants droit ou l'assuré) devront communiquer à la Société tous renseignements utiles concernant le sinistre (Ancienne Mutuelle Vie). Sinistre, cependant, est un terme assez général, et qui ne convient pas dans tous les cas. On le trouve au niveau des traités, des statistiques, de la régie interne: Death claim, Sinistre mortuaire (et non réclamation de décès); Claims admitted, Sinistres reconnus (et non réclamations admises); Claims paid, Sinistres réglés (et non réclamations payées). Il revient aussi très fréquemment dans les contrats, mais à peu près jamais dans les polices d'assurance-vie, où on lui préfère le mot décès.

Cette tendance à ne pas évoquer l'idée de sinistre dans les contrats-vie est généralisée (et compréhensible !) mais il est assez facile de respecter les convenances: If there is no claim, Si le risque ne se réalise pas $(A M V)$ - Provided there has been no claim under paragraph 2, Pourvu que l'assuré n'ait pas bénéficié des prestations prévues à l'alinéa 2 - Ce droit de révocation ne peut être exercé qu'après l'exigibilité de la somme assurée (Loi du 13 juillet 1930).

Ouvrons une première parenthèse pour dire que les auteurs rendent le mot happening par survenance: Il est donc tout à fait normal que l'assureur ne conserve pas (la réserve) après la survenance d'un sinistre qu'il refuse de prendre en charge (Maurice Fauque); et une deuxième pour nous demander si l'expression contrat sinistré, qui a cours en France, n'est pas fautive. René Georgin condamne en effet les tandems du genre assurés sociaux, prévention routière, dans lesquels la caractérisation est en porte-à-faux. En effet, c'est l'assurance qui est sociale et non l'assuré, 
et l'épithète routière s'applique aux accidents de la route et non à la prévention. Dans notre cas, si le contrat était sinistré, la compagnie le remplacerait volontiers.

\section{DEUXIÈME SENS}

Dans les assurances sur la vie, lorsqu'il correspond à la demande de la somme stipulée au contrat, claim peut se traduire par demande de règlement. La plupart du temps, on peut retrancher le déterminé: Les pièces à remettre à la compagnie pour le règlement sont ... (L'Union) - Before payment of any claim under this policy, Avant le règlement des sommes assurées par la (présente) police - Claims department, Service des règlements (et non département des réclamations).

Dans les autres branches d'assurance, on dit demande de prestations, demande d'indemnités, demande d'indemnisation: Toute demande d'indemnités doit être accompagnée d'un certificat médical (Assurance-invalidité, Providentia de Genève). L'on peut aussi rendre claim en utilisant le seul déterminant du composé demande d'indemnités: Les parties s'interdisent d'avoir recours à la voie judiciaire pour le règlement des indemnités (L'Union). Il est aussi possible de transposer vers le participe: Lorsqu'une prestation est demandée pour une invalidité partielle (Providentia). Claims department devient ici service des sinistres.

Claim désigne aussi la somme à verser. Payment of claim, paiement des sommes assurées, des prestations, des indemnités (et non paiement de la réclamation). Le paiement des sommes dues est indivisible à l'égard de la société (L'Union).

On notera, dans les alinéas qui précèdent et dans ceux qui suivent, le caractère analytique du français, qui multiplie les expressions pour mieux préciser la pensée. Le procédé cause parfois une amplification, mais permet aussi un gain d'information.

Dans les polices de France que j'ai en ma possession, je n'ai pas trouvé d'expressions commodes pour traduire proof of claim, que nous traduisons au Canada par preuve de réclamation. Mais j'ai relevé dans cinq ou six polices de la Suisse le terme justification des prétentions. Prétention est en effet l'un des sens de claim: To lay a claim to something, Prétendre à quelque chose (Th. A. Quemner, Dictionnaire juridique) - To renounce one's claim, Renoncer à ses prétentions (Jules Jéraute, Vocabulaire de termes et locutions juridiques). Justification, qui semble plus abstrait que preuve, témoigne sans doute de l'affinité du français pour le plan de l'entendement et l'on pourrait y voir l'un des gages de l'authenticité de l'expression en question. Voici deux exemples: Si l'assuré refuse de se soumettre à un examen médical, la prétention est réputée non fondée (Providentia) - S'il y a eu lors de la prétention aux prestations ... dol ou réticence de la part de l'ayant droit (Ibid.) - Admission of claim, Reconnaissance des prétentions (et non admission de la réclamation). En France, où la réalité a peut-être été découpée un peu différemment, on semble préférer le mot preuve que l'on accole à un déterminant spécifique: La preuve de l'invalidité incombe aux sociétaires (Ancienne Mutuelle Vie). Ajoutons ici que notice of claim se traduit par déclaration (d'invalidité, d'accident, d'incapacité de travail, etc.) et non par avis de réclamation.

\section{TROISIÈME SENS}

Celui de droit à l'assurance, aux sommes assurées, aux prestations, à l'indemnisation. À la rubrique claim, Koessler et Derocquigny relèvent le sens de droit, 
de titre, qui est celui de claim dans les phrases suivantes: a) He makes claim for the amount of money which the company has promised to pay; b) Claims might be denied after the policy was issued. Traduction proposée: a) Il fait valoir ses droits à la somme que la compagnie a promis de lui verser; b) Une fois la police souscrite, il se peut que l'on refuse de faire droit à une demande (d'indemnité). Le mot droit a aussi le sens de claim dans cette dernière citation: L'emploi intentionnel de documents inexacts, ou de moyens frauduleux entraîne ... la déchéance de tous les droits à l'indemnité (Ancienne Mutuelle Accidents).

\section{QUATRIÈME SENS}

Celui de revendication, de recours, de réclamation. Dans les textes d'assurance de personnes, il est assez rarement question de claim dans cette acception. En voici tout de même un exemple: All benefits payable under this policy will be exempt and free from claims of the owner's creditors. Ajoutons deux citations tirées du Code des Assurances de France, dans lesquelles réclamation correspond sans doute à claim: Bien entendu, la réclamation de la victime peut être faite à l'assuré ou à l'assureur - Ce risque ne sera réalisé que par la réclamation amiable ou judiciaire ... du tiers lésé. Enfin, cette phrase extraite du même livre: L'assureur pouvait obtenir la répétition des indemnités de sinistres payés pour un manquement quelconque de l'assuré dans ses déclarations, où répétition a le sens de claiming back.

Réclamant - Si la condamnation de réclamer et réclamation s'impose, il faut naturellement s'élever aussi contre réclamant, dont l'emploi est également injustifié. Il est d'ailleurs facile de lui substituer d'autres termes honorables: ayant droit, intéressé (dans sa forme nominale) ou encore, personne. Le paiement des sommes dues est indivisible à l'égard de la Société, qui règle sur quittance conjointe des intéressés - The identity of the claimant entitled to settlement, L'identité de l'ayant droit (Économie appréciable !) - When there are two or more claimants for the same proceeds, Lorsqu'une ou plusieurs personnes réclament (on insiste sans doute) la même somme assurée.

Disons pour conclure qu'en langue correcte, un ayant droit n'a pas à réclamer une somme assurée: il y a contradiction dans les termes. Si l'on veut corriger cette errevir, il faudra sans doute rompre avec bien des habitudes de langue, et renoncer au calque. La liste des termes traités dans cet article est incomplète. Espérons tout de même que cette brève étude permettra aux traducteurs et aux rédacteurs de résoudre plus facilement les problèmes connexes qu'ils rencontreront au hasard de leurs travaux et d'apporter dans ce domaine leur appoint à l'effort de normalisation de la langue.

Nous nous réjouissons à l'idée que les préposés à la rédaction de la nouvelle loi des assurances feront eux aussi cet effort (Ce sera la pierre au fond du sac qui retiendra sous l'eau Raminagrobis!) et que leurs textes nous serviront de « haute référence en matière de bon langage $»$. Il faudra particulièrement qu'ils fassent état de l'impropriété du mot réclamation. Ce terme-omnibus ne correspond pas aux réalités de l'assurance. De plus, l'emploi que l'on en a fait jusqu'à maintenant s'inspire d'un synthétisme dont l'anglais s'accommode sans doute, mais qui répugne au caractère analytique de notre langue.

JeAN-PAUl de GrandPré 


\section{À PROPOS DE "GIGGLE"}

Le traducteur se trouve parfois embarrassé pour rendre, avec la même expressivité, l'anglais « to giggle » ou l'allemand « kichern ».

Ainsi, nous relevons les traductions suivantes:

LD (allemand) : «Die Mädchen kicherten $~$.

LA (anglais): «The young girls giggled 》.

LA (français) : «Les jeunes filles étouffèrent un petit rire».

$\mathrm{Si}$ nous consultons le dictionnaire Sachs-Villatte ${ }^{2}$, nous trouvons pour « kichern »: rire d'un rire étouffé, rire sous cape, rioter (langage familier). Dans le dictionnaire Harrap ${ }^{3}$, nous trouvons pour « giggle »: rire nerveusement, bêtement, avec affectation, glousser: «pousser des petits rires». D'un autre côté, le dictionnaire Larousse ${ }^{4}$ donne pour rioter: rire un peu: «Il ne fait que rioter ».

$\mathrm{Ni}$ le grand Larousse encyclopédique ${ }^{5}$, ni le Robert ${ }^{6}$ n'enregistrent le terme. Mais nous trouvons le mot dans le Dictionnaire des synonymes ${ }^{7}$ avec l'explication suivante: «Rioter, vx, rire à demi, souvent dédaigneusement ». « Rioter» ne semble donc pas convenir pour rendre «to giggle » ou «kichern ».

Nous avons cependant trouvé dans l'Extrême-Occident $^{8}$ la phrase suivante: "Il y avait de la grâce dans son visage de vieil adolescent, avec ce long nez un tout petit peu de travers et ce sourire de garçonnet: c'était un des rares hommes capables de riocher comme les gamins et fillettes, ce que les Anglais appellent to giggle et les Allemands kichern. »

Le seul dictionnaire qui donne le terme riocher est le Littré ${ }^{9}$. Même si ce mot a vieilli, il nous semble cependant possible de lui injecter une nouvelle jeunesse: l'expressivité phonétique n'aura plus rien à envier à to giggle ou à kichern.

Traducteurs, à vous de décider!

ANDRÉ Clas

\section{EN ALUMINIUM - D'ALUMINIUM QUESTION}

Dans les expressions déterminant la matière dont une chose est faite, faut-il se servir de en ou de de? Exemples: Une poutrelle d'acier ou en acier; un piston d'aluminium ou en aluminium.

1. Heinrich Böll, Billiard um halb zehn, Cologne, Kiepenheuer et Witsch, 1959, p. 246. Trad. angl.: Billiards at Half-Past-Nine, Patrick Bowles, trad., New York, McGraw-Hill, 1962. Trad. franç.: les Deux Sacrements, S. et G. De Lalène, trad., Paris, Seuil, 1960.

2. Sachs-Villatte, Enzyklopädisches Wörterbuch der französischen und deutschen Sprache, $30^{\mathrm{e}}$ éd., 2 vol., Berlin, Langenscheidt, 1954.

3. J.E. Mansion, éd., Harrap's Standard French and English Dictionary, 2 vol., Londres, Harrap, 1961.

4. Larousse du XX $X^{e}$ siècle, 6 vol., Paris, Larousse, 1934.

5. Grand Larousse encyclopédique, 10 vol., Paris, Larousse, 1964.

6. Paul Robert, éd,, Dictionnaire alphabétique et analogique de la langue française, Paris, Société du nouveau Littré, 1964

7. Henri Bénac, Dictionnaire des synonymes, Paris, Hachette, 1956.

8. Petru Dumitriu, l'Extrême-Occident, Paris, Seuil, 1964.

9. E. Littré, éd., Dictionnaire de la langue française, 4 vol., Paris, Hachette, 1874. 